\title{
Intercriteria analysis of countries in transition from factor-driven to efficiency-driven economy
}

\author{
Vassia Atanassova $^{1}$, Lyubka Doukovska ${ }^{2}$ and Maciej Krawczak ${ }^{3}$ \\ ${ }^{1}$ Bioinformatics and Mathematical Modelling Department \\ Institute of Biophysics and Biomedical Engineering, Bulgarian Academy of Sciences \\ 105 Acad. Georgi Bonchev Str., Sofia 1113, Bulgaria \\ e-mail: vassia.atanassova@gmail.com \\ ${ }^{2}$ Intelligent Systems Department \\ Institute of Information and Communication Technologies, Bulgarian Academy of Sciences \\ 2 Acad. Georgi Bonchev Str., Sofia 1113, Bulgaria \\ e-mail: doukovska@iit.bas.bg \\ ${ }^{3}$ Systems Research Institute, Polish Academy of Sciences, and \\ Warsaw School of Information Technology \\ 6 Newelska Str., Warsaw, Poland \\ e-mail: krawczak@ibspan.waw.pl
}

Received: 28 March 2018

Accepted: 20 April 2018

\begin{abstract}
The intuitionistic fuzzy sets-based method of InterCriteria Analysis is applied here to datasets retrieved from the World Economic Forum's Global Competitiveness Reports (GCRs) from years 2013-2014 to 2017-2018 containing global economies whose stage of development is in the transition from factor-driven to efficiency-driven economy. These data are analysed in search of correlations between the twelve pillars of competitiveness and certain findings are outlined and commented.
\end{abstract}

Keywords: InterCriteria Analysis, Intuitionistic fuzzy sets, Factor-driven economy, Efficiencydriven economy, Competitiveness, Economic development, Knowledge discovery.

2010 Mathematic Subject Classification: 03E72.

\section{Introduction}

The present paper is a subsequent step in the research of application of the intuitionistic fuzzy sets-based method of InterCriteria Analysis of the performance of global economies against the 
pillars of competitiveness in the methodology of the World Economic Forum. Previous applications of the method were over data for the countries in the efficiency-driven stage, [11] and those in transition from efficiency to innovation-driven economies, [8]. Here our focus of investigation are the economies in the transition phase between factor-driven and efficiencydriven economies (Stage 1 to Stage 2). The analysed datasets are from the 5-year period from year 2013-2014 to year 2017-2018 and are derived from the publicly available Global Competitiveness Report of the World Economic Forum, [18-22].

According to the World Economic Forum, the well-known economic theory of stages of development postulates that in the first stage, the economy is factor-driven and countries compete based on their factor endowments - primarily unskilled labor and natural resources. Companies compete on the basis of price and sell basic products or commodities, with their low productivity reflected in low wages, [22, p. 319]. The factors of competitiveness at this stage of development relies to the greatest extent on well-functioning public and private institutions (pillar 1), a well-developed infrastructure (pillar 2), a stable macroeconomic environment (pillar 3), and workforce that has received at least a basic education and healthcare (pillar 4). Factor-driven economies are highly sensitive to world economic cycles, commodity prices, and exchange rate fluctuations, mitigated only in countries with large internal market to attract investment independent of export potential.

Transition of an economy to efficiency-driven stage of development takes place as it becomes more competitive, with increased and more-efficient productivity, higher product quality, advancing development and rising wages. At this point, competitiveness is increasingly driven by higher education and training (5th pillar), efficient goods markets (6th pillar), well-functioning labor markets (7th pillar), developed financial markets (8th pillar), the ability to harness the benefits of existing technologies (9th pillar), and a large domestic or foreign market (10th pillar), [22, p. 319].

In the methodology, it is considered that all countries that export more than 70 percent of mineral products are considered to be to some extent factor driven. In countries that export less than 70 percent minerals, stages of development are dictated uniquely by income. [22, p. 323]. The methodology of WEF employs the following weights in order to determine the belonging of a country to a stage (see Table 1).

\begin{tabular}{|l|c|c|c|c|c|}
\cline { 2 - 6 } \multicolumn{1}{c|}{} & \multicolumn{5}{c|}{ STAGE OF DEVELOPMENT } \\
\cline { 2 - 6 } \multicolumn{1}{c|}{} & $\begin{array}{c}\text { Stage 1: } \\
\text { Factor- } \\
\text { driven }\end{array}$ & $\begin{array}{c}\text { Transition } \\
\text { from Stage 1 } \\
\text { to Stage 2 }\end{array}$ & $\begin{array}{c}\text { Stage 2: } \\
\text { Efficiency- } \\
\text { driven }\end{array}$ & $\begin{array}{c}\text { Transition } \\
\text { from Stage 2 } \\
\text { to Stage 3 }\end{array}$ & $\begin{array}{c}\text { Stage 3: } \\
\text { Innovation- } \\
\text { driven }\end{array}$ \\
\hline GDP per capita (US\$) thresholds* & $<2,000$ & $2,000-2,999$ & $3,000-8,999$ & $9,000-17,000$ & $>17,000$ \\
\hline Weight for basic requirements & $60 \%$ & $40-60 \%$ & $40 \%$ & $20-40 \%$ & $20 \%$ \\
\hline Weight for efficiency enhancers & $35 \%$ & $35-50 \%$ & $50 \%$ & $50 \%$ & $50 \%$ \\
\hline $\begin{array}{l}\text { Weight for innovation and } \\
\text { sophistication factors }\end{array}$ & $5 \%$ & $5-10 \%$ & $10 \%$ & $10-30 \%$ & $30 \%$ \\
\hline
\end{tabular}

Table 1. Subindex weights and income thresholds for stages of development, [22, p. 320] 


\section{Presentation of the input data}

In the investigated 5-year period, the countries that comprised the set of factor-to-efficiency driven economies has been repeatedly the smallest set of all the five sets of factor-driven economies (Stage 1), efficiency-driven economies (Stage 2), innovation-driven economies (Stage 3), and the two transition stages in between. Over the years, the set has the following appearing elements, as given in details in Table 2.

\begin{tabular}{|c|c|c|c|c|c|}
\hline 2013-2014 & 2014-2015 & 2015-2016 & 2016-2017 & 2017-2018 & $\begin{array}{l}\text { Total \# } \\
\text { appear. }\end{array}$ \\
\hline Algeria & Algeria & Algeria & Algeria & Algeria & 5 \\
\hline Angola & Angola & & & & 2 \\
\hline Armenia & & & & & 1 \\
\hline Azerbaijan & Azerbaijan & Azerbaijan & Azerbaijan & Azerbaijan & 5 \\
\hline Bhutan & Bhutan & Bhutan & Bhutan & Bhutan & 5 \\
\hline Bolivia & Bolivia & & Bolivia & & 3 \\
\hline Botswana & Botswana & Botswana & Botswana & Botswana & 5 \\
\hline Brunei Darussalam & & & Brunei Darussalam & Brunei Darussalam & 3 \\
\hline Gabon & Gabon & Gabon & Gabon & & 4 \\
\hline Honduras & Honduras & Honduras & Honduras & Honduras & 5 \\
\hline \multirow[t]{2}{*}{ Iran, Islamic Rep } & Iran, Islamic Rep & Iran, Islamic Rep & & & 3 \\
\hline & & Kazakhstan & Kazakhstan & Kazakhstan & 3 \\
\hline Kuwait & Kuwait & Kuwait & Kuwait & Kuwait & 5 \\
\hline Libya & Libya & & & & 2 \\
\hline Moldova & Moldova & Moldova & & & 3 \\
\hline Mongolia & Mongolia & Mongolia & Mongolia & Mongolia & 5 \\
\hline \multirow[t]{3}{*}{ Morocco } & & & & & 1 \\
\hline & & & & Nicaragua & 1 \\
\hline & & Nigeria & Nigeria & Nigeria & 3 \\
\hline \multirow[t]{2}{*}{ Philippines } & Philippines & Philippines & Philippines & Philippines & 5 \\
\hline & & & Russian Federation & & 1 \\
\hline Saudi Arabia & Saudi Arabia & Saudi Arabia & & & 3 \\
\hline \multirow[t]{2}{*}{ Sri Lanka } & & & & & 1 \\
\hline & & & Ukraine & Ukraine & 2 \\
\hline \multirow[t]{2}{*}{ Venezuela } & Venezuela & Venezuela & Venezuela & Venezuela & 5 \\
\hline & & Vietnam & Vietnam & Vietnam & 3 \\
\hline 20 & 16 & 16 & 17 & 15 & \\
\hline
\end{tabular}

Table 2. Lists of factor-to-innovation driven economies in the investigated 5-year period

As we can see, the set of countries being analysed is interesting in that it contains some constant members, true representatives of this economic transition, but also countries which sporadically enter this group, or leave it over the years. Hence, the belongingness of the elements (countries) to the set depends on their performance according to the criteria within the predefined limits, which is a substancial difference from working with a set of fixed elements, 
which belongingness does not depend on their performance, as in the case of analyzing the relations between the pillars of competitivess of EU28 member states, see e.g. [7, 9, 10].

Another aspect we need to remark on here is the relatively low number of objects for analysis, compared to related applications of ICA, for instance over the data for the efficiencydriven and innovation-driven economies in the world, which are about twice more, or some other applications of ICA with hundreds or even thousands numbers of objects. While the question of representability is irrelevant here, as these 15-20 objects are all possible elements of the set, exclusively and in the way it has been defined, we still shall mention that with a lower number of objects, the results cannot be considered as robust as in cases when ICA is applied on datasets with much bigger number of objects, as it has been shown in [24].

The input data, derived from the GCRs, represents the evaluation of these sets of countries (in ICA terms: objects) against the 12 pillars of competitiveness (in ICA terms: criteria), with the evaluations being numbers on the 1-to-7 scale with decimal precision of 0.1 , where the 12 pillars of competitiveness have been formed on the basis of more than 110 subindicators derived from the countries' national statistics. The input datasets for the investigated years, from 2013-2014 to 2017-2018, are given in the following Tables 3 to 7.

\begin{tabular}{|c|c|c|c|c|c|c|c|c|c|c|c|c|c|c|c|c|c|c|c|c|}
\hline $\begin{array}{l}\text { Factor-to-efficiency } \\
\text { 2013-2014 }\end{array}$ & $\frac{-\frac{\pi}{2}}{\frac{0}{0}}$ & $\frac{\pi}{\frac{\pi}{0}}$ & 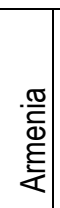 & 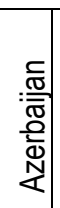 & 苞 & $\frac{. \infty}{\bar{D}}$ & 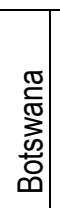 & 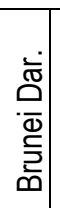 & 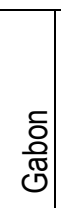 & 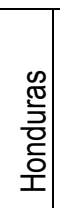 & 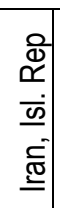 & | & 発 & $\begin{array}{l}\frac{0}{3} \\
\text { 음 } \\
\frac{0}{2}\end{array}$ & $\begin{array}{l}\frac{.0}{\overline{0}} \\
\frac{1}{2} \\
\frac{0}{2}\end{array}$ & 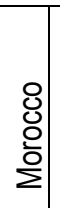 & 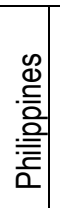 & 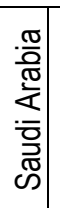 & 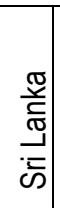 & $\begin{array}{l}\frac{\mathbb{d}}{\mathbb{N}} \\
\mathbf{N} \\
\mathbb{d} \\
\stackrel{\mathbb{N}}{>}\end{array}$ \\
\hline 1 Institutions & 3.0 & 2.8 & 4.0 & 4.1 & 4.4 & 3.4 & 4.7 & 5.0 & 3.7 & 3.1 & 3.7 & 4.2 & 3.2 & 3.2 & 3.3 & 4.1 & 3.8 & 5.1 & 4.1 & 2.3 \\
\hline 2 Infrastructure & 3.1 & 1.9 & 3.8 & 4.1 & 3.6 & 3.0 & 3.4 & 4.3 & 2.8 & 2.8 & 4.1 & 4.4 & 3.2 & 3.6 & 2.9 & 4.3 & 3.4 & 5.2 & 4.0 & 2.6 \\
\hline 3 Macroeconomic environment & 5.5 & 5.0 & 4.9 & 6.4 & 4.1 & 5.7 & 5.8 & 7.0 & 6.1 & 4.3 & 4.3 & 6.7 & 6.0 & 4.6 & 3.7 & 4.4 & 5.3 & 6.7 & 3.9 & 3.1 \\
\hline 4 Health and primary education & 5.4 & 3.7 & 5.5 & 5.1 & 5.4 & 5.1 & 4.6 & 6.3 & 4.1 & 5.4 & 6.0 & 5.6 & 4.5 & 5.4 & 5.6 & 5.5 & 5.3 & 5.9 & 5.9 & 5.5 \\
\hline 5 Higher education and training & 3.5 & 2.1 & 4.2 & 4.0 & 3.4 & 3.8 & 3.6 & 4.5 & 2.6 & 3.3 & 4.0 & 4.0 & 3.5 & 3.9 & 4.1 & 3.5 & 4.3 & 4.7 & 4.3 & 4.2 \\
\hline 6 Goods market efficiency & 3.2 & 3.0 & 4.3 & 4.3 & 3.9 & 3.5 & 4.1 & 4.5 & 3.6 & 3.9 & 3.9 & 4.1 & 3.1 & 3.9 & 4.1 & 4.3 & 4.2 & 4.8 & 4.6 & 2.8 \\
\hline 7 Labor market efficiency & 2.9 & 3.7 & 4.5 & 4.7 & 4.7 & 3.7 & 4.5 & 5.1 & 4.3 & 3.3 & 3.0 & 4.0 & 3.5 & 4.1 & 4.5 & 3.9 & 4.1 & 4.3 & 3.5 & 2.8 \\
\hline 8 Financial market development & 2.6 & 2.4 & 3.9 & 3.8 & 3.3 & 3.4 & 4.3 & 4.3 & 3.6 & 4.2 & 3.2 & 4.0 & 2.3 & 3.6 & 3.2 & 4.0 & 4.4 & 4.7 & 4.5 & 3.0 \\
\hline 9 Technological readiness & 2.5 & 2.5 & 3.7 & 4.2 & 2.6 & 2.8 & 3.1 & 3.8 & 3.0 & 3.1 & 3.0 & 3.8 & 2.7 & 3.9 & 3.8 & 3.5 & 3.6 & 4.6 & 3.3 & 3.1 \\
\hline 10 Market size & 4.4 & 3.8 & 2.7 & 3.6 & 1.8 & 3.3 & 3.0 & 2.4 & 2.7 & 3.2 & 5.1 & 3.8 & 3.5 & 2.5 & 2.7 & 4.2 & 4.7 & 5.1 & 3.9 & 4.6 \\
\hline 11 Business sophistication & 2.9 & 2.9 & 3.8 & 4.0 & 3.5 & 3.6 & 3.6 & 4.2 & 3.0 & 3.8 & 3.6 & 3.9 & 3.2 & 3.3 & 3.3 & 3.7 & 4.3 & 4.7 & 4.5 & 3.2 \\
\hline 12 Innovation & 2.4 & 2.1 & 3.0 & 3.5 & 2.8 & 3.2 & 3.0 & 3.4 & 2.5 & 2.8 & 3.2 & 2.8 & 2.2 & 2.4 & 2.9 & 2.9 & 3.2 & 3.9 & 3.5 & 2.5 \\
\hline
\end{tabular}

Table 3. Factor-to-efficiency economies in 2013-2014 (Source: [18]).

\begin{tabular}{|c|c|c|c|c|c|c|c|c|c|c|c|c|c|c|c|c|}
\hline $\begin{array}{c}\text { Factor-to-efficiency } \\
2014-2015\end{array}$ & $\begin{array}{l}\frac{\pi}{\frac{0}{2}} \\
\frac{\operatorname{m}}{2}\end{array}$ & $\frac{\pi}{\circ}$ & 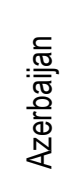 & 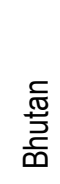 & $\stackrel{\cdot \frac{\pi}{2}}{\stackrel{\bar{O}}{\infty}}$ & 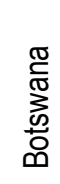 & 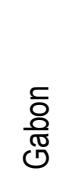 & 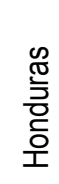 & 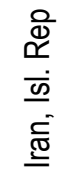 & 产 & 莺 & $\begin{array}{l}\frac{\pi}{2} \\
\frac{0}{0} \\
\frac{0}{2}\end{array}$ & $\begin{array}{l}\frac{-\pi}{\bar{O}} \\
\bar{D} \\
\text { D }\end{array}$ & 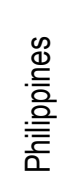 & 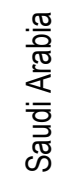 & $\begin{array}{l}\frac{\mathbb{N}}{\mathbb{N}} \\
\stackrel{N}{\mathbb{N}} \\
\stackrel{\mathbb{N}}{>}\end{array}$ \\
\hline 1 Institutions & 3.4 & 2.6 & 4 & 4.5 & 3.5 & 4.5 & 3.7 & 3.4 & 3.4 & 4.0 & 2.6 & 3.2 & 3.4 & 3.9 & 5.0 & 2.1 \\
\hline 2 Infrastructure & 3.1 & 2.0 & 4.1 & 3.5 & 3.0 & 3.2 & 2.9 & 3.2 & 4.1 & 4.3 & 2.9 & 3.7 & 2.9 & 3.5 & 5.2 & 2.6 \\
\hline 3 Macroeconomic environment & 6.4 & 4.7 & 6.4 & 3.9 & 5.5 & 6.3 & 6.0 & 3.8 & 4.8 & 6.7 & 5.4 & 4.9 & 3.8 & 5.8 & 6.7 & 3.1 \\
\hline 4 Health and primary education & 5.6 & 3.5 & 5.2 & 5.5 & 4.9 & 4.1 & 4.0 & 5.5 & 6.0 & 5.6 & 4.6 & 5.4 & 5.8 & 5.4 & 6.0 & 5.5 \\
\hline 5 Higher education and training & 3.7 & 1.9 & 3.9 & 3.5 & 3.7 & 3.6 & 2.8 & 3.6 & 4.2 & 4.2 & 3.6 & 4.1 & 4.4 & 4.4 & 4.6 & 4.3 \\
\hline
\end{tabular}




\begin{tabular}{|l|c|c|c|c|c|c|c|c|c|c|c|c|c|c|c|c|}
\hline 6 Goods market efficiency & 3.5 & 2.9 & 4.3 & 4.0 & 3.6 & 4.1 & 3.8 & 4.2 & 3.9 & 4.0 & 3.3 & 4.1 & 4.2 & 4.3 & 4.7 & 2.8 \\
\hline 7 Labor market efficiency & 3.1 & 3.5 & 4.6 & 4.7 & 3.6 & 4.6 & 4.2 & 3.5 & 3.0 & 3.8 & 3.4 & 4.1 & 4.5 & 4.0 & 4.2 & 2.6 \\
\hline 8 Financial market development & 2.7 & 2.5 & 3.8 & 3.4 & 3.3 & 4.2 & 3.6 & 4.2 & 3.0 & 3.9 & 1.9 & 3.7 & 3.2 & 4.4 & 4.7 & 2.9 \\
\hline 9 Technological readiness & 2.6 & 2.3 & 4.3 & 2.7 & 2.8 & 3.6 & 3.0 & 3.2 & 3.0 & 3.7 & 2.6 & 4.4 & 3.5 & 3.8 & 4.5 & 3.0 \\
\hline 10 Market size & 4.4 & 3.8 & 3.7 & 1.8 & 3.4 & 3.1 & 2.9 & 3.2 & 5.1 & 3.8 & 3.3 & 2.6 & 2.7 & 4.7 & 5.1 & 4.6 \\
\hline 11 Business sophistication & 3.2 & 2.6 & 3.9 & 3.6 & 3.6 & 3.5 & 3.2 & 4.0 & 3.5 & 3.9 & 3.0 & 3.4 & 3.5 & 4.3 & 4.6 & 3.0 \\
\hline 12 Innovation & 2.6 & 2.1 & 3.3 & 2.9 & 3.1 & 3 & 2.7 & 3.2 & 3.1 & 2.9 & 2.0 & 2.5 & 2.9 & 3.5 & 3.8 & 2.4 \\
\hline
\end{tabular}

Table 4. Factor-to-efficiency economies in 2014-2015 (Source: [19]).

\begin{tabular}{|c|c|c|c|c|c|c|c|c|c|c|c|c|c|c|c|c|}
\hline $\begin{array}{l}\text { Factor-to-efficiency } \\
\text { 2015-2016 }\end{array}$ & $\frac{\cdot \frac{0}{\frac{0}{2}}}{\frac{0}{2}}$ & 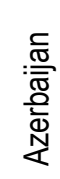 & 㽕 & 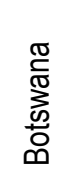 & $\begin{array}{l}\text { ర్ } \\
\text { ్ㅠ }\end{array}$ & 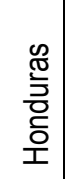 & 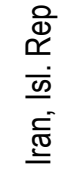 & 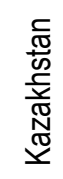 & 党 & $\frac{\text { 임 }}{\frac{0}{0}}$ & $\begin{array}{l}\frac{.00}{\bar{D}} \\
\text { ㅁ } \\
\text { 을 }\end{array}$ & $\frac{-\frac{\pi}{2}}{\frac{0}{2}}$ & 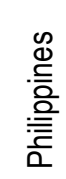 & 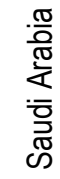 & $\begin{array}{l}\frac{\mathbb{N}}{\mathbb{N}} \\
\mathbf{N} \\
\frac{\mathbb{N}}{\mathbb{N}} \\
>\end{array}$ & 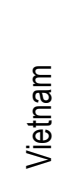 \\
\hline 1 Institutions & 3.5 & 3.9 & 4.6 & 4.4 & 3.8 & 3.6 & 3.6 & 4.2 & 4.1 & 3.2 & 3.6 & 3.2 & 3.8 & 5.1 & 2.1 & 3.7 \\
\hline 2 Infrastructure & 3.1 & 4.1 & 3.4 & 3.3 & 2.9 & 3.4 & 4.2 & 4.2 & 4.3 & 3.7 & 2.9 & 2.1 & 3.4 & 5.1 & 2.6 & 3.8 \\
\hline 3 Macroeconomic environment & 5.3 & 6.4 & 3.6 & 6.5 & 6.0 & 4.1 & 4.8 & 5.7 & 6.7 & 4.9 & 3.2 & 4.6 & 5.7 & 6.6 & 2.9 & 4.7 \\
\hline 4 Health and primary education & 5.6 & 5.2 & 5.4 & 4.5 & 4.7 & 5.4 & 6.0 & 5.4 & 5.6 & 5.4 & 5.7 & 2.9 & 5.5 & 6.0 & 5.5 & 5.9 \\
\hline 5 Higher education and training & 3.7 & 3.9 & 3.6 & 3.7 & 2.8 & 3.8 & 4.3 & 4.5 & 4.0 & 4.1 & 4.5 & 2.8 & 4.5 & 4.7 & 4.5 & 3.8 \\
\hline 6 Goods market efficiency & 3.5 & 4.3 & 4.0 & 4.1 & 3.8 & 4.3 & 4.0 & 4.5 & 4.1 & 4.1 & 4.2 & 4.1 & 4.2 & 4.7 & 2.8 & 4.2 \\
\hline 7 Labor market efficiency & 3.2 & 4.6 & 4.8 & 4.5 & 4.2 & 3.7 & 3.2 & 4.9 & 3.7 & 4.1 & 4.5 & 4.5 & 4.1 & 4.3 & 2.6 & 4.4 \\
\hline 8 Financial market development & 2.8 & 3.3 & 3.6 & 4.0 & 3.5 & 4.4 & 2.8 & 3.6 & 3.8 & 3.3 & 3.0 & 3.8 & 4.2 & 4.3 & 2.8 & 3.7 \\
\hline 9 Technological readiness & 2.6 & 4.3 & 2.9 & 3.3 & 2.9 & 3.2 & 3.2 & 4.2 & 4.3 & 4.4 & 4.0 & 3.0 & 3.9 & 4.7 & 3.1 & 3.3 \\
\hline 10 Market size & 4.7 & 3.9 & 1.8 & 3.0 & 2.9 & 3.1 & 5.2 & 4.5 & 4.2 & 2.7 & 3.0 & 5.1 & 4.9 & 5.4 & 4.7 & 4.8 \\
\hline 11 Business sophistication & 3.3 & 3.9 & 3.6 & 3.5 & 3.2 & 4.1 & 3.5 & 3.8 & 4.0 & 3.3 & 3.5 & 3.7 & 4.3 & 4.5 & 3.0 & 3.6 \\
\hline 12 Innovation & 2.8 & 3.3 & 2.9 & 3.0 & 2.6 & 3.4 & 3.1 & 3.3 & 3.0 & 2.6 & 3.1 & 2.8 & 3.5 & 3.8 & 2.4 & 3.2 \\
\hline
\end{tabular}

Table 5. Factor-to-efficiency economies in 2015-2016 (Source: [20]).

\begin{tabular}{|c|c|c|c|c|c|c|c|c|c|c|c|c|c|c|c|c|c|}
\hline $\begin{array}{l}\text { Factor-to-efficiency } \\
\text { 2016-2017 }\end{array}$ & $\begin{array}{l}\frac{\pi}{\frac{\pi}{2}} \\
\frac{0}{\frac{0}{2}}\end{array}$ & $\begin{array}{l}\frac{c}{\mathbb{0}} \\
: \frac{1}{\mathbb{N}} \\
\frac{0}{\overline{0}} \\
\frac{N}{4}\end{array}$ & $\begin{array}{l}\frac{c}{\mathbb{N}} \\
\frac{\bar{T}}{\infty}\end{array}$ & 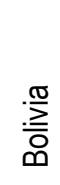 & 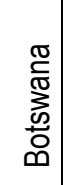 & 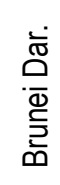 & $\begin{array}{l}\bar{D} \\
\text { ్ㅠ } \\
\mathbb{0}\end{array}$ & 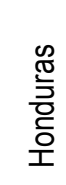 & 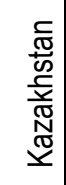 & 䓠 & 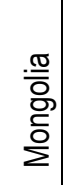 & $\frac{\frac{\pi}{2}}{\frac{\pi}{2}}$ & 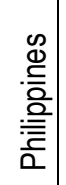 & 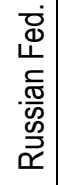 & 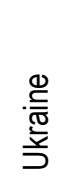 & 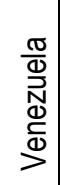 & 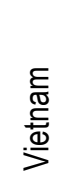 \\
\hline 1 Institutions & 3.5 & 4.2 & 4.7 & 2.9 & 4.5 & 4.2 & 3.7 & 3.3 & 4.2 & 4.1 & 3.5 & 3.3 & 3.6 & 3.6 & 3.0 & 2.2 & 3.8 \\
\hline 2 Infrastructure & 3.3 & 4.3 & 3.4 & 3.2 & 3.5 & 3.9 & 3.1 & 3.3 & 4.2 & 4.4 & 2.9 & 2.1 & 3.4 & 4.9 & 3.9 & 2.6 & 3.9 \\
\hline 3 Macroeconomic environment & 4.8 & 5.2 & 3.8 & 4.0 & 6.2 & 4.9 & 5.6 & 4.9 & 4.7 & 6.3 & 3.6 & 4.0 & 5.9 & 4.3 & 3.2 & 2.4 & 4.5 \\
\hline 4 Health and primary education & 5.7 & 5.7 & 5.3 & 5.3 & 4.7 & 6.3 & 4.8 & 5.6 & 5.4 & 5.6 & 5.7 & 2.8 & 5.6 & 5.9 & 6.0 & 5.4 & 5.8 \\
\hline 5 Higher education and training & 3.9 & 4.2 & 3.8 & 3.8 & 4.1 & 4.5 & 3.0 & 3.6 & 4.6 & 4.0 & 4.6 & 2.9 & 4.6 & 5.1 & 5.1 & 4.7 & 4.1 \\
\hline 6 Goods market efficiency & 3.5 & 4.5 & 4.0 & 3.4 & 4.3 & 4.3 & 3.7 & 4.1 & 4.4 & 4.2 & 4.0 & 4.1 & 4.1 & 4.2 & 4.0 & 2.9 & 4.2 \\
\hline 7 Labor market efficiency & 3.2 & 4.8 & 4.7 & 3.1 & 4.5 & 4.5 & 3.9 & 3.6 & 4.8 & 3.7 & 4.3 & 4.5 & 4.0 & 4.4 & 4.2 & 2.8 & 4.3 \\
\hline 8 Financial market development & 2.9 & 3.5 & 3.9 & 3.9 & 4.0 & 3.7 & 3.5 & 4.5 & 3.5 & 4.0 & 3.1 & 3.7 & 4.2 & 3.4 & 3.0 & 3.1 & 3.9 \\
\hline 9 Technological readiness & 3.1 & 4.5 & 3.2 & 3.0 & 3.6 & 3.6 & 3.1 & 3.1 & 4.4 & 4.3 & 4.1 & 3.1 & 3.6 & 4.3 & 3.6 & 3.1 & 3.5 \\
\hline 10 Market size & 4.7 & 3.9 & 1.8 & 3.4 & 2.9 & 2.7 & 2.8 & 3.1 & 4.5 & 4.3 & 2.9 & 5.0 & 4.9 & 5.9 & 4.4 & 4.6 & 4.8 \\
\hline 11 Business sophistication & 3.3 & 4.0 & 3.7 & 3.2 & 3.6 & 3.7 & 3.2 & 3.8 & 3.6 & 4.0 & 3.3 & 3.6 & 4.1 & 3.8 & 3.6 & 3.0 & 3.6 \\
\hline 12 Innovation & 2.9 & 3.6 & 3.1 & 2.5 & 3.2 & 3.3 & 2.7 & 3.1 & 3.4 & 3.0 & 3.1 & 2.9 & 3.4 & 3.4 & 3.4 & 2.6 & 3.3 \\
\hline
\end{tabular}

Table 6. Factor-to-efficiency economies in 2016-2017(Source: [21]). 


\begin{tabular}{|c|c|c|c|c|c|c|c|c|c|c|c|c|c|c|c|}
\hline 2017-2018 & 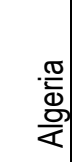 & 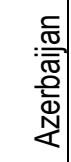 & $\begin{array}{l}\frac{c}{\mathbb{m}} \\
\stackrel{\vec{D}}{\bar{m}}\end{array}$ & 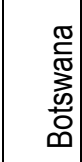 & 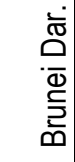 & $\begin{array}{l}\mathscr{2} \\
\stackrel{\mathbb{T}}{=} \\
\overline{0} \\
\text { 오 }\end{array}$ & 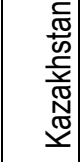 & 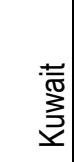 & $\begin{array}{l}\frac{-\pi}{\bar{O}} \\
\mathrm{D} \\
\frac{0}{2}\end{array}$ & $\begin{array}{l}\mathbb{N} \\
\mathbb{0} \\
\frac{\mathbb{N}}{\mathbb{N}} \\
\frac{\mathbb{U}}{Z}\end{array}$ & $\begin{array}{l}-\frac{\pi}{\bar{d}} \\
\frac{0}{\bar{z}}\end{array}$ & 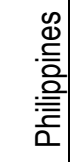 & 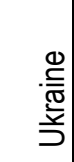 & $\begin{array}{l}\frac{\mathbb{N}}{\mathbb{D}} \\
\mathbf{N} \\
\mathbb{D} \\
\mathbb{D} \\
>\end{array}$ & 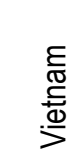 \\
\hline 1 Institutions & 3.6 & 4.6 & 4.8 & 4.4 & 4.4 & 3.2 & 4.0 & 4.0 & 3.4 & 3.2 & 3.2 & 3.5 & 3.2 & 2.2 & 3.8 \\
\hline 2 Infrastructure & 3.6 & 4.5 & 3.6 & 3.6 & 4.3 & 3.2 & 4.2 & 4.3 & 3.1 & 3.6 & 2.0 & 3.4 & 3.9 & 2.6 & 3.9 \\
\hline 3 Macroeconomic environment & 4.6 & 4.8 & 4.6 & 6.1 & 5.1 & 5.0 & 4.2 & 5.6 & 4.4 & 5.1 & 3.5 & 5.8 & 3.5 & 2.4 & 4.6 \\
\hline 4 Health and primary education & 5.8 & 5.7 & 5.4 & 4.8 & 6.3 & 5.5 & 5.9 & 5.6 & 5.6 & 5.5 & 3.0 & 5.6 & 6.0 & 5.3 & 5.8 \\
\hline 5 Higher education and training & 4.0 & 4.5 & 4.0 & 3.8 & 4.5 & 3.6 & 4.6 & 3.9 & 4.5 & 3.4 & 3.1 & 4.6 & 5.1 & 4.6 & 4.1 \\
\hline 6 Goods market efficiency & 3.6 & 4.8 & 4.2 & 4.2 & 4.3 & 4.0 & 4.3 & 4.2 & 4.0 & 3.9 & 4.1 & 4.0 & 4.0 & 2.8 & 4.1 \\
\hline 7 Labor market efficiency & 3.3 & 5.0 & 4.7 & 4.5 & 4.4 & 3.5 & 4.6 & 3.6 & 4.2 & 3.9 & 4.6 & 4.0 & 4.0 & 2.7 & 4.3 \\
\hline 8 Financial market development & 3.1 & 3.8 & 4.0 & 4.0 & 3.7 & 4.5 & 3.3 & 4.1 & 3.0 & 3.6 & 3.7 & 4.2 & 3.1 & 3.1 & 4.0 \\
\hline 9 Technological readiness & 3.4 & 4.6 & 3.2 & 3.6 & 4.5 & 3.3 & 4.6 & 4.3 & 4.2 & 3.1 & 3.0 & 3.8 & 3.8 & 3.0 & 4.0 \\
\hline 10 Market size & 4.8 & 4.0 & 1.9 & 3.0 & 2.9 & 3.1 & 4.5 & 4.4 & 3.0 & 3.0 & 5.0 & 5.0 & 4.5 & 4.4 & 4.9 \\
\hline 11 Business sophistication & 3.3 & 4.4 & 3.8 & 3.7 & 3.7 & 3.8 & 3.6 & 4.0 & 3.3 & 3.2 & 3.7 & 4.1 & 3.7 & 3.0 & 3.7 \\
\hline 12 Innovation & 2.9 & 4.0 & 3.2 & 3.2 & 3.2 & 2.9 & 3.2 & 3.0 & 3.0 & 2.5 & 2.8 & 3.3 & 3.4 & 2.6 & 3.3 \\
\hline
\end{tabular}

Table 7. Factor-to-efficiency economies in 2017-2018 (Source: [22]).

\section{Results and discussion}

Here we presume that the reader is already familiar with the InterCriteria Analysis and we are not going to present it in details. The theory of ICA has been presented in $[1-3,5,12,16,17$, 23]. We will only remind here that from each input dataset of evaluations of objects (in this case, countries) against criteria (i.e., pillars of competitiveness), ICA computes a new table that contains intuitionistic fuzzy pairs, [4], giving the measure of dependence between every pair of criteria. In ICA-specific terms, the IFP gives dependence between two criteria falls in one of the three possible categories: positive consonance, negative consonance or dissonance. Positive consonance in ICA is interpreted as the definite presence of relation between two criteria, with boundary value of $\langle 1 ; 0\rangle$, negative consonance is interpreted as the definite absence of relation, with boundary value $\langle 0 ; 1\rangle$; and dissonance is interpreted as uncertainty, where no particular conclusion can be derived, with boundary value $\langle 0 ; 0\rangle$. Since every criterion perfectly correlates with itself, the IFPs along the main diagonal are all equal to $\langle 1 ; 0\rangle$, and the intercriteria dependence is a commutative property, i.e. $\left\langle\mu_{C i, C k} ; v_{C i, C k}\right\rangle=\left\langle\mu_{C k, C i} ; v_{C k, C i}\right\rangle$.

The computations are performed with the two developed ICA software applications [1315], which for the sake of simplicity return the computed result in the form of two tables, one giving the membership parts of the IF pairs, and the other giving the non-membership parts. Therefore, here we present the results of the application of ICA in tabular way by two tables per year with the membership and non-membership parts of the intercriteria pairs (Tables 8 a) and b) to 12 a) and b)), and in graphic way as points plotted on the intuitionistic fuzzy interpretational triangle, [6] (Figure 1, a) to d)). On this basis the findings are commented as follows. 


\begin{tabular}{|l|c|c|c|c|c|c|c|c|c|c|c|c|}
\hline \multicolumn{1}{|c|}{ a) Membership } & 1 & 2 & 3 & 4 & 5 & 6 & 7 & 8 & 9 & 10 & 11 & 12 \\
\hline 1 Institutions & 1.000 & 0.779 & 0.642 & 0.579 & 0.621 & 0.779 & 0.721 & 0.747 & 0.653 & 0.421 & 0.732 & 0.716 \\
\hline 2 Infrastructure & 0.779 & 1.000 & 0.600 & 0.695 & 0.674 & 0.747 & 0.600 & 0.679 & 0.679 & 0.526 & 0.737 & 0.679 \\
\hline 3 Macroeconomic environment & 0.642 & 0.600 & 1.000 & 0.374 & 0.500 & 0.547 & 0.616 & 0.584 & 0.558 & 0.474 & 0.568 & 0.537 \\
\hline 4 Health and primary education & 0.579 & 0.695 & 0.374 & 1.000 & 0.716 & 0.674 & 0.447 & 0.584 & 0.626 & 0.521 & 0.642 & 0.637 \\
\hline 5 Higher education and training & 0.621 & 0.674 & 0.500 & 0.716 & 1.000 & 0.700 & 0.532 & 0.647 & 0.700 & 0.558 & 0.711 & 0.721 \\
\hline 6 Goods market efficiency & 0.779 & 0.747 & 0.547 & 0.674 & 0.700 & 1.000 & 0.653 & 0.779 & 0.726 & 0.442 & 0.795 & 0.768 \\
\hline 7 Labor market efficiency & 0.721 & 0.600 & 0.616 & 0.447 & 0.532 & 0.653 & 1.000 & 0.589 & 0.642 & 0.263 & 0.584 & 0.579 \\
\hline 8 Financial market development & 0.747 & 0.679 & 0.584 & 0.584 & 0.647 & 0.779 & 0.589 & 1.000 & 0.679 & 0.511 & 0.821 & 0.732 \\
\hline 9 Technological readiness & 0.653 & 0.679 & 0.558 & 0.626 & 0.700 & 0.726 & 0.642 & 0.679 & 1.000 & 0.442 & 0.726 & 0.647 \\
\hline 10 Market size & 0.421 & 0.526 & 0.474 & 0.521 & 0.558 & 0.442 & 0.263 & 0.511 & 0.442 & 1.000 & 0.532 & 0.532 \\
\hline 11 Business sophistication & 0.732 & 0.737 & 0.568 & 0.642 & 0.711 & 0.795 & 0.584 & 0.821 & 0.726 & 0.532 & 1.000 & 0.789 \\
\hline 12 Innovation & 0.716 & 0.679 & 0.537 & 0.637 & 0.721 & 0.768 & 0.579 & 0.732 & 0.647 & 0.532 & 0.789 & 1.000 \\
\hline
\end{tabular}

\begin{tabular}{|l|c|c|c|c|c|c|c|c|c|c|c|c|}
\hline \multicolumn{1}{|c|}{ b) Non-membership } & 1 & 2 & 3 & 4 & 5 & 6 & 7 & 8 & 9 & 10 & 11 & 12 \\
\hline 1 Institutions & 0.000 & 0.168 & 0.321 & 0.332 & 0.311 & 0.142 & 0.211 & 0.205 & 0.289 & 0.526 & 0.205 & 0.211 \\
\hline 2 Infrastructure & 0.168 & 0.000 & 0.363 & 0.226 & 0.268 & 0.174 & 0.332 & 0.274 & 0.253 & 0.421 & 0.200 & 0.237 \\
\hline 3 Macroeconomic environment & 0.321 & 0.363 & 0.000 & 0.553 & 0.447 & 0.389 & 0.332 & 0.384 & 0.389 & 0.489 & 0.384 & 0.395 \\
\hline 4 Health and primary education & 0.332 & 0.226 & 0.553 & 0.000 & 0.189 & 0.253 & 0.447 & 0.332 & 0.279 & 0.389 & 0.258 & 0.263 \\
\hline 5 Higher education and training & 0.311 & 0.268 & 0.447 & 0.189 & 0.000 & 0.195 & 0.384 & 0.289 & 0.216 & 0.374 & 0.211 & 0.179 \\
\hline 6 Goods market efficiency & 0.142 & 0.174 & 0.389 & 0.253 & 0.195 & 0.000 & 0.253 & 0.137 & 0.179 & 0.468 & 0.105 & 0.121 \\
\hline 7 Labor market efficiency & 0.211 & 0.332 & 0.332 & 0.447 & 0.384 & 0.253 & 0.000 & 0.347 & 0.274 & 0.679 & 0.337 & 0.332 \\
\hline 8 Financial market development & 0.205 & 0.274 & 0.384 & 0.332 & 0.289 & 0.137 & 0.347 & 0.000 & 0.258 & 0.442 & 0.121 & 0.189 \\
\hline 9 Technological readiness & 0.289 & 0.253 & 0.389 & 0.279 & 0.216 & 0.179 & 0.274 & 0.258 & 0.000 & 0.489 & 0.205 & 0.253 \\
\hline 10 Market size & 0.526 & 0.421 & 0.489 & 0.389 & 0.374 & 0.468 & 0.679 & 0.442 & 0.489 & 0.000 & 0.405 & 0.384 \\
\hline 11 Business sophistication & 0.205 & 0.200 & 0.384 & 0.258 & 0.211 & 0.105 & 0.337 & 0.121 & 0.205 & 0.405 & 0.000 & 0.126 \\
\hline 12 Innovation & 0.211 & 0.237 & 0.395 & 0.263 & 0.179 & 0.121 & 0.332 & 0.189 & 0.253 & 0.384 & 0.126 & 0.000 \\
\hline
\end{tabular}

Table 8. Results of ICA on the data of factor-to-efficiency economies in 2013-2014.

\begin{tabular}{|l|c|c|c|c|c|c|c|c|c|c|c|c|}
\hline \multicolumn{1}{|c|}{ a) Membership } & 1 & 2 & 3 & 4 & 5 & 6 & 7 & 8 & 9 & 10 & 11 & 12 \\
\hline 1 Institutions & 1.000 & 0.675 & 0.700 & 0.492 & 0.475 & 0.717 & 0.725 & 0.742 & 0.617 & 0.442 & 0.725 & 0.708 \\
\hline 2 Infrastructure & 0.675 & 1.000 & 0.667 & 0.633 & 0.633 & 0.708 & 0.575 & 0.733 & 0.717 & 0.550 & 0.742 & 0.700 \\
\hline 3 Macroeconomic environment & 0.700 & 0.667 & 1.000 & 0.458 & 0.517 & 0.592 & 0.583 & 0.642 & 0.600 & 0.600 & 0.583 & 0.583 \\
\hline 4 Health and primary education & 0.492 & 0.633 & 0.458 & 1.000 & 0.733 & 0.567 & 0.417 & 0.517 & 0.558 & 0.617 & 0.600 & 0.592 \\
\hline 5 Higher education and training & 0.475 & 0.633 & 0.517 & 0.733 & 1.000 & 0.633 & 0.433 & 0.600 & 0.692 & 0.675 & 0.617 & 0.617 \\
\hline 6 Goods market efficiency & 0.717 & 0.708 & 0.592 & 0.567 & 0.633 & 1.000 & 0.692 & 0.817 & 0.792 & 0.458 & 0.792 & 0.792 \\
\hline 7 Labor market efficiency & 0.725 & 0.575 & 0.583 & 0.417 & 0.433 & 0.692 & 1.000 & 0.650 & 0.633 & 0.283 & 0.600 & 0.600 \\
\hline 8 Financial market development & 0.742 & 0.733 & 0.642 & 0.517 & 0.600 & 0.817 & 0.650 & 1.000 & 0.783 & 0.525 & 0.817 & 0.783 \\
\hline 9 Technological readiness & 0.617 & 0.717 & 0.600 & 0.558 & 0.692 & 0.792 & 0.633 & 0.783 & 1.000 & 0.500 & 0.700 & 0.700 \\
\hline 10 Market size & 0.442 & 0.550 & 0.600 & 0.617 & 0.675 & 0.458 & 0.283 & 0.525 & 0.500 & 1.000 & 0.525 & 0.592 \\
\hline 11 Business sophistication & 0.725 & 0.742 & 0.583 & 0.600 & 0.617 & 0.792 & 0.600 & 0.817 & 0.700 & 0.525 & 1.000 & 0.833 \\
\hline 12 Innovation & 0.708 & 0.700 & 0.583 & 0.592 & 0.617 & 0.792 & 0.600 & 0.783 & 0.700 & 0.592 & 0.833 & 1.000 \\
\hline
\end{tabular}




\begin{tabular}{|l|c|c|c|c|c|c|c|c|c|c|c|c|}
\hline \multicolumn{1}{|c|}{ b) Non-membership } & 1 & 2 & 3 & 4 & 5 & 6 & 7 & 8 & 9 & 10 & 11 & 12 \\
\hline 1 Institutions & 0.000 & 0.200 & 0.217 & 0.383 & 0.400 & 0.192 & 0.175 & 0.175 & 0.275 & 0.467 & 0.175 & 0.183 \\
\hline 2 Infrastructure & 0.200 & 0.000 & 0.258 & 0.267 & 0.283 & 0.208 & 0.350 & 0.225 & 0.200 & 0.383 & 0.150 & 0.217 \\
\hline 3 Macroeconomic environment & 0.217 & 0.258 & 0.000 & 0.467 & 0.408 & 0.367 & 0.367 & 0.325 & 0.342 & 0.358 & 0.333 & 0.358 \\
\hline 4 Health and primary education & 0.383 & 0.267 & 0.467 & 0.000 & 0.167 & 0.350 & 0.508 & 0.425 & 0.358 & 0.333 & 0.292 & 0.325 \\
\hline 5 Higher education and training & 0.400 & 0.283 & 0.408 & 0.167 & 0.000 & 0.283 & 0.492 & 0.358 & 0.225 & 0.258 & 0.275 & 0.300 \\
\hline 6 Goods market efficiency & 0.192 & 0.208 & 0.367 & 0.350 & 0.283 & 0.000 & 0.250 & 0.142 & 0.142 & 0.492 & 0.117 & 0.158 \\
\hline 7 Labor market efficiency & 0.175 & 0.350 & 0.367 & 0.508 & 0.492 & 0.250 & 0.000 & 0.317 & 0.308 & 0.675 & 0.317 & 0.342 \\
\hline 8 Financial market development & 0.175 & 0.225 & 0.325 & 0.425 & 0.358 & 0.142 & 0.317 & 0.000 & 0.175 & 0.450 & 0.117 & 0.175 \\
\hline 9 Technological readiness & 0.275 & 0.200 & 0.342 & 0.358 & 0.225 & 0.142 & 0.308 & 0.175 & 0.000 & 0.450 & 0.208 & 0.233 \\
\hline 10 Market size & 0.467 & 0.383 & 0.358 & 0.333 & 0.258 & 0.492 & 0.675 & 0.450 & 0.450 & 0.000 & 0.400 & 0.358 \\
\hline 11 Business sophistication & 0.175 & 0.150 & 0.333 & 0.292 & 0.275 & 0.117 & 0.317 & 0.117 & 0.208 & 0.400 & 0.000 & 0.075 \\
\hline 12 Innovation & 0.183 & 0.217 & 0.358 & 0.325 & 0.300 & 0.158 & 0.342 & 0.175 & 0.233 & 0.358 & 0.075 & 0.000 \\
\hline
\end{tabular}

Table 9. Results of ICA on the data of factor-to-efficiency economies in 2014-2015.

\begin{tabular}{|l|c|c|c|c|c|c|c|c|c|c|c|c|}
\hline \multicolumn{1}{|c|}{ a) Membership } & 1 & 2 & 3 & 4 & 5 & 6 & 7 & 8 & 9 & 10 & 11 & 12 \\
\hline 1 Institutions & 1.000 & 0.667 & 0.717 & 0.442 & 0.467 & 0.608 & 0.650 & 0.633 & 0.600 & 0.433 & 0.658 & 0.658 \\
\hline 2 Infrastructure & 0.667 & 1.000 & 0.675 & 0.617 & 0.625 & 0.642 & 0.492 & 0.542 & 0.700 & 0.558 & 0.675 & 0.667 \\
\hline 3 Macroeconomic environment & 0.717 & 0.675 & 1.000 & 0.417 & 0.492 & 0.550 & 0.500 & 0.592 & 0.650 & 0.525 & 0.600 & 0.575 \\
\hline 4 Health and primary education & 0.442 & 0.617 & 0.417 & 1.000 & 0.658 & 0.450 & 0.300 & 0.383 & 0.517 & 0.633 & 0.492 & 0.567 \\
\hline 5 Higher education and training & 0.467 & 0.625 & 0.492 & 0.658 & 1.000 & 0.583 & 0.383 & 0.417 & 0.675 & 0.600 & 0.542 & 0.625 \\
\hline 6 Goods market efficiency & 0.608 & 0.642 & 0.550 & 0.450 & 0.583 & 1.000 & 0.617 & 0.658 & 0.700 & 0.517 & 0.733 & 0.783 \\
\hline 7 Labor market efficiency & 0.650 & 0.492 & 0.500 & 0.300 & 0.383 & 0.617 & 1.000 & 0.533 & 0.542 & 0.383 & 0.575 & 0.550 \\
\hline 8 Financial market development & 0.633 & 0.542 & 0.592 & 0.383 & 0.417 & 0.658 & 0.533 & 1.000 & 0.558 & 0.517 & 0.733 & 0.658 \\
\hline 9 Technological readiness & 0.600 & 0.700 & 0.650 & 0.517 & 0.675 & 0.700 & 0.542 & 0.558 & 1.000 & 0.492 & 0.642 & 0.642 \\
\hline 10 Market size & 0.433 & 0.558 & 0.525 & 0.633 & 0.600 & 0.517 & 0.383 & 0.517 & 0.492 & 1.000 & 0.600 & 0.608 \\
\hline 11 Business sophistication & 0.658 & 0.675 & 0.600 & 0.492 & 0.542 & 0.733 & 0.575 & 0.733 & 0.642 & 0.600 & 1.000 & 0.825 \\
\hline 12 Innovation & 0.658 & 0.667 & 0.575 & 0.567 & 0.625 & 0.783 & 0.550 & 0.658 & 0.642 & 0.608 & 0.825 & 1.000 \\
\hline
\end{tabular}

\begin{tabular}{|l|c|c|c|c|c|c|c|c|c|c|c|c|}
\hline \multicolumn{1}{|c|}{ b) Non-membership } & 1 & 2 & 3 & 4 & 5 & 6 & 7 & 8 & 9 & 10 & 11 & 12 \\
\hline 1 Institutions & 0.000 & 0.250 & 0.233 & 0.442 & 0.417 & 0.275 & 0.258 & 0.275 & 0.342 & 0.508 & 0.275 & 0.275 \\
\hline 2 Infrastructure & 0.250 & 0.000 & 0.275 & 0.283 & 0.258 & 0.225 & 0.417 & 0.367 & 0.225 & 0.383 & 0.242 & 0.250 \\
\hline 3 Macroeconomic environment & 0.233 & 0.275 & 0.000 & 0.500 & 0.442 & 0.350 & 0.442 & 0.350 & 0.308 & 0.450 & 0.350 & 0.375 \\
\hline 4 Health and primary education & 0.442 & 0.283 & 0.500 & 0.000 & 0.208 & 0.383 & 0.575 & 0.508 & 0.375 & 0.275 & 0.392 & 0.317 \\
\hline 5 Higher education and training & 0.417 & 0.258 & 0.442 & 0.208 & 0.000 & 0.267 & 0.492 & 0.458 & 0.217 & 0.308 & 0.342 & 0.258 \\
\hline 6 Goods market efficiency & 0.275 & 0.225 & 0.350 & 0.383 & 0.267 & 0.000 & 0.258 & 0.217 & 0.175 & 0.375 & 0.133 & 0.100 \\
\hline 7 Labor market efficiency & 0.258 & 0.417 & 0.442 & 0.575 & 0.492 & 0.258 & 0.000 & 0.383 & 0.375 & 0.567 & 0.350 & 0.358 \\
\hline 8 Financial market development & 0.275 & 0.367 & 0.350 & 0.508 & 0.458 & 0.217 & 0.383 & 0.000 & 0.358 & 0.433 & 0.175 & 0.250 \\
\hline 9 Technological readiness & 0.342 & 0.225 & 0.308 & 0.375 & 0.217 & 0.175 & 0.375 & 0.358 & 0.000 & 0.458 & 0.283 & 0.283 \\
\hline 10 Market size & 0.508 & 0.383 & 0.450 & 0.275 & 0.308 & 0.375 & 0.567 & 0.433 & 0.458 & 0.000 & 0.358 & 0.333 \\
\hline 11 Business sophistication & 0.275 & 0.242 & 0.350 & 0.392 & 0.342 & 0.133 & 0.350 & 0.175 & 0.283 & 0.358 & 0.000 & 0.108 \\
\hline 12 Innovation & 0.275 & 0.250 & 0.375 & 0.317 & 0.258 & 0.100 & 0.358 & 0.250 & 0.283 & 0.333 & 0.108 & 0.000 \\
\hline
\end{tabular}

Table 10. Results of ICA on the data of factor-to-efficiency economies in 2015-2016. 


\begin{tabular}{|l|c|c|c|c|c|c|c|c|c|c|c|c|}
\hline \multicolumn{1}{|c|}{ a) Membership } & 1 & 2 & 3 & 4 & 5 & 6 & 7 & 8 & 9 & 10 & 11 & 12 \\
\hline 1 Institutions & 1.000 & 0.640 & 0.669 & 0.449 & 0.434 & 0.728 & 0.735 & 0.566 & 0.618 & 0.324 & 0.588 & 0.596 \\
\hline 2 Infrastructure & 0.640 & 1.000 & 0.610 & 0.632 & 0.625 & 0.706 & 0.625 & 0.463 & 0.728 & 0.515 & 0.662 & 0.735 \\
\hline 3 Macroeconomic environment & 0.669 & 0.610 & 1.000 & 0.412 & 0.368 & 0.654 & 0.522 & 0.640 & 0.529 & 0.441 & 0.625 & 0.507 \\
\hline 4 Health and primary education & 0.449 & 0.632 & 0.412 & 1.000 & 0.676 & 0.522 & 0.485 & 0.338 & 0.544 & 0.529 & 0.522 & 0.610 \\
\hline 5 Higher education and training & 0.434 & 0.625 & 0.368 & 0.676 & 1.000 & 0.507 & 0.500 & 0.301 & 0.625 & 0.588 & 0.478 & 0.684 \\
\hline 6 Goods market efficiency & 0.728 & 0.706 & 0.654 & 0.522 & 0.507 & 1.000 & 0.757 & 0.522 & 0.721 & 0.471 & 0.647 & 0.713 \\
\hline 7 Labor market efficiency & 0.735 & 0.625 & 0.522 & 0.485 & 0.500 & 0.757 & 1.000 & 0.478 & 0.647 & 0.434 & 0.588 & 0.669 \\
\hline 8 Financial market development & 0.566 & 0.463 & 0.640 & 0.338 & 0.301 & 0.522 & 0.478 & 1.000 & 0.390 & 0.390 & 0.588 & 0.426 \\
\hline 9 Technological readiness & 0.618 & 0.728 & 0.529 & 0.544 & 0.625 & 0.721 & 0.647 & 0.390 & 1.000 & 0.456 & 0.588 & 0.721 \\
\hline 10 Market size & 0.324 & 0.515 & 0.441 & 0.529 & 0.588 & 0.471 & 0.434 & 0.390 & 0.456 & 1.000 & 0.485 & 0.507 \\
\hline 11 Business sophistication & 0.588 & 0.662 & 0.625 & 0.522 & 0.478 & 0.647 & 0.588 & 0.588 & 0.588 & 0.485 & 1.000 & 0.654 \\
\hline 12 Innovation & 0.550 & 0.733 & 0.483 & 0.592 & 0.675 & 0.683 & 0.633 & 0.450 & 0.683 & 0.492 & 0.625 & 1.000 \\
\hline
\end{tabular}

\begin{tabular}{|l|c|c|c|c|c|c|c|c|c|c|c|c|}
\hline \multicolumn{1}{|c|}{ b) Non-membership } & 1 & 2 & 3 & 4 & 5 & 6 & 7 & 8 & 9 & 10 & 11 & 12 \\
\hline 1 Institutions & 0.000 & 0.279 & 0.272 & 0.463 & 0.478 & 0.169 & 0.199 & 0.338 & 0.228 & 0.625 & 0.272 & 0.294 \\
\hline 2 Infrastructure & 0.279 & 0.000 & 0.338 & 0.272 & 0.294 & 0.184 & 0.301 & 0.434 & 0.140 & 0.441 & 0.206 & 0.162 \\
\hline 3 Macroeconomic environment & 0.272 & 0.338 & 0.000 & 0.515 & 0.574 & 0.257 & 0.426 & 0.279 & 0.331 & 0.537 & 0.250 & 0.397 \\
\hline 4 Health and primary education & 0.463 & 0.272 & 0.515 & 0.000 & 0.235 & 0.360 & 0.419 & 0.551 & 0.272 & 0.404 & 0.324 & 0.250 \\
\hline 5 Higher education and training & 0.478 & 0.294 & 0.574 & 0.235 & 0.000 & 0.375 & 0.419 & 0.603 & 0.206 & 0.360 & 0.382 & 0.221 \\
\hline 6 Goods market efficiency & 0.169 & 0.184 & 0.257 & 0.360 & 0.375 & 0.000 & 0.147 & 0.338 & 0.125 & 0.449 & 0.169 & 0.147 \\
\hline 7 Labor market efficiency & 0.199 & 0.301 & 0.426 & 0.419 & 0.419 & 0.147 & 0.000 & 0.449 & 0.206 & 0.522 & 0.279 & 0.213 \\
\hline 8 Financial market development & 0.338 & 0.434 & 0.279 & 0.551 & 0.603 & 0.338 & 0.449 & 0.000 & 0.419 & 0.537 & 0.235 & 0.426 \\
\hline 9 Technological readiness & 0.228 & 0.140 & 0.331 & 0.272 & 0.206 & 0.125 & 0.206 & 0.419 & 0.000 & 0.412 & 0.191 & 0.103 \\
\hline 10 Market size & 0.625 & 0.441 & 0.537 & 0.404 & 0.360 & 0.449 & 0.522 & 0.537 & 0.412 & 0.000 & 0.397 & 0.404 \\
\hline 11 Business sophistication & 0.272 & 0.206 & 0.250 & 0.324 & 0.382 & 0.169 & 0.279 & 0.235 & 0.191 & 0.397 & 0.000 & 0.169 \\
\hline 12 Innovation & 0.325 & 0.150 & 0.417 & 0.258 & 0.225 & 0.158 & 0.233 & 0.400 & 0.117 & 0.408 & 0.183 & 0.000 \\
\hline
\end{tabular}

Table 11. Results of ICA on the data of factor-to-efficiency economies in 2016-2017.

\begin{tabular}{|l|c|c|c|c|c|c|c|c|c|c|c|c|}
\hline \multicolumn{1}{|c|}{ a) Membership } & 1 & 2 & 3 & 4 & 5 & 6 & 7 & 8 & 9 & 10 & 11 & 12 \\
\hline 1 Institutions & 1.000 & 0.640 & 0.669 & 0.449 & 0.434 & 0.728 & 0.735 & 0.566 & 0.618 & 0.324 & 0.588 & 0.596 \\
\hline 2 Infrastructure & 0.640 & 1.000 & 0.610 & 0.632 & 0.625 & 0.706 & 0.625 & 0.463 & 0.728 & 0.515 & 0.662 & 0.735 \\
\hline 3 Macroeconomic environment & 0.669 & 0.610 & 1.000 & 0.412 & 0.368 & 0.654 & 0.522 & 0.640 & 0.529 & 0.441 & 0.625 & 0.507 \\
\hline 4 Health and primary education & 0.449 & 0.632 & 0.412 & 1.000 & 0.676 & 0.522 & 0.485 & 0.338 & 0.544 & 0.529 & 0.522 & 0.610 \\
\hline 5 Higher education and training & 0.434 & 0.625 & 0.368 & 0.676 & 1.000 & 0.507 & 0.500 & 0.301 & 0.625 & 0.588 & 0.478 & 0.684 \\
\hline 6 Goods market efficiency & 0.728 & 0.706 & 0.654 & 0.522 & 0.507 & 1.000 & 0.757 & 0.522 & 0.721 & 0.471 & 0.647 & 0.713 \\
\hline 7 Labor market efficiency & 0.735 & 0.625 & 0.522 & 0.485 & 0.500 & 0.757 & 1.000 & 0.478 & 0.647 & 0.434 & 0.588 & 0.669 \\
\hline 8 Financial market development & 0.566 & 0.463 & 0.640 & 0.338 & 0.301 & 0.522 & 0.478 & 1.000 & 0.390 & 0.390 & 0.588 & 0.426 \\
\hline 9 Technological readiness & 0.618 & 0.728 & 0.529 & 0.544 & 0.625 & 0.721 & 0.647 & 0.390 & 1.000 & 0.456 & 0.588 & 0.721 \\
\hline 10 Market size & 0.324 & 0.515 & 0.441 & 0.529 & 0.588 & 0.471 & 0.434 & 0.390 & 0.456 & 1.000 & 0.485 & 0.507 \\
\hline 11 Business sophistication & 0.588 & 0.662 & 0.625 & 0.522 & 0.478 & 0.647 & 0.588 & 0.588 & 0.588 & 0.485 & 1.000 & 0.654 \\
\hline 12 Innovation & 0.550 & 0.733 & 0.483 & 0.592 & 0.675 & 0.683 & 0.633 & 0.450 & 0.683 & 0.492 & 0.625 & 1.000 \\
\hline
\end{tabular}




\begin{tabular}{|l|c|c|c|c|c|c|c|c|c|c|c|c|}
\hline \multicolumn{1}{|c|}{ b) Non-membership } & 1 & 2 & 3 & 4 & 5 & 6 & 7 & 8 & 9 & 10 & 11 & 12 \\
\hline 1 Institutions & 0.000 & 0.162 & 0.286 & 0.333 & 0.429 & 0.114 & 0.200 & 0.324 & 0.219 & 0.581 & 0.229 & 0.219 \\
\hline 2 Infrastructure & 0.162 & 0.000 & 0.333 & 0.190 & 0.343 & 0.171 & 0.333 & 0.400 & 0.152 & 0.514 & 0.276 & 0.219 \\
\hline 3 Macroeconomic environment & 0.286 & 0.333 & 0.000 & 0.495 & 0.581 & 0.333 & 0.438 & 0.219 & 0.400 & 0.543 & 0.267 & 0.371 \\
\hline 4 Health and primary education & 0.333 & 0.190 & 0.495 & 0.000 & 0.229 & 0.343 & 0.438 & 0.524 & 0.190 & 0.400 & 0.371 & 0.257 \\
\hline 5 Higher education and training & 0.429 & 0.343 & 0.581 & 0.229 & 0.000 & 0.400 & 0.476 & 0.571 & 0.267 & 0.362 & 0.419 & 0.210 \\
\hline 6 Goods market efficiency & 0.114 & 0.171 & 0.333 & 0.343 & 0.400 & 0.000 & 0.114 & 0.324 & 0.181 & 0.495 & 0.200 & 0.219 \\
\hline 7 Labor market efficiency & 0.200 & 0.333 & 0.438 & 0.438 & 0.476 & 0.114 & 0.000 & 0.429 & 0.333 & 0.524 & 0.286 & 0.267 \\
\hline 8 Financial market development & 0.324 & 0.400 & 0.219 & 0.524 & 0.571 & 0.324 & 0.429 & 0.000 & 0.448 & 0.438 & 0.133 & 0.362 \\
\hline 9 Technological readiness & 0.219 & 0.152 & 0.400 & 0.190 & 0.267 & 0.181 & 0.333 & 0.448 & 0.000 & 0.476 & 0.305 & 0.200 \\
\hline 10 Market size & 0.581 & 0.514 & 0.543 & 0.400 & 0.362 & 0.495 & 0.524 & 0.438 & 0.476 & 0.000 & 0.390 & 0.410 \\
\hline 11 Business sophistication & 0.229 & 0.276 & 0.267 & 0.371 & 0.419 & 0.200 & 0.286 & 0.133 & 0.305 & 0.390 & 0.000 & 0.181 \\
\hline 12 Innovation & 0.220 & 0.231 & 0.385 & 0.231 & 0.198 & 0.220 & 0.264 & 0.374 & 0.209 & 0.418 & 0.187 & 0.000 \\
\hline
\end{tabular}

Table 12. Results of ICA on the data of factor-to-efficiency economies in 2017-2018.

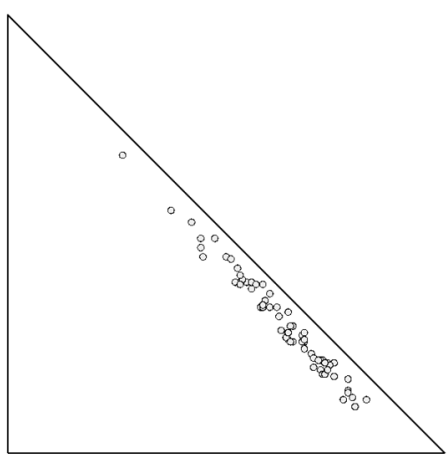

a)

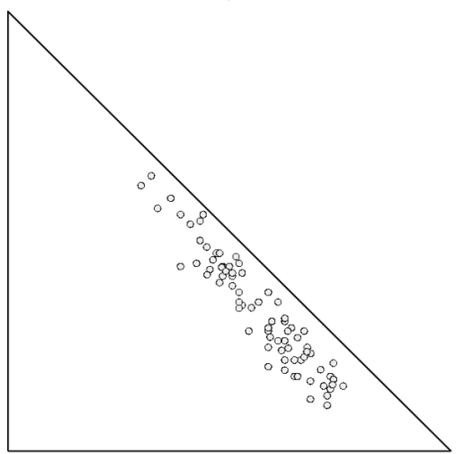

d)

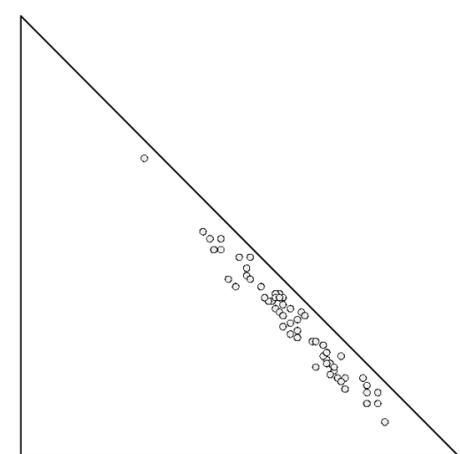

b)

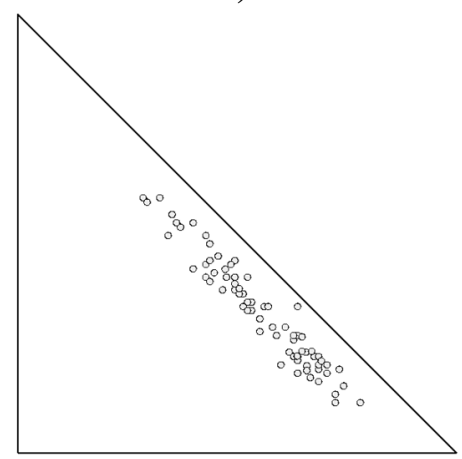

e)

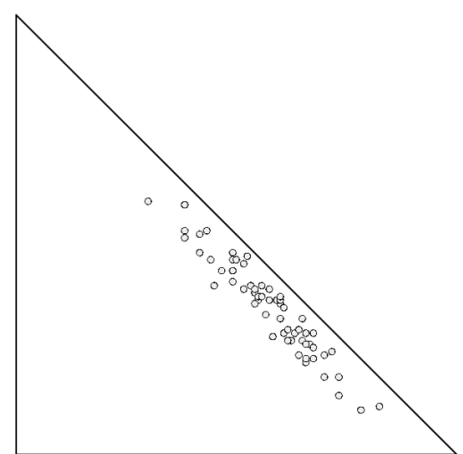

c)

Figure 1. Graphic interpretation of the results of application of ICA on the data of factor-toefficiency economies:

a) in year 2013-2014;

b) in year 2014-2015;

c) in year 2015-2016;

d) in year 2016-2017;

e) in year 2017-2018.

In the results over the years we notice the strong and persistent presence of pillar "6 Goods market efficiency", with high positive consonances with pillar "8 Financial market development", "9 Technological readiness" and "12 Innovation". In some years, two of the traditionally lowest correlating pillars, the basic requirement "3 Macroeconomic environment" (2015-2016) and the economy enhancer "10 Market size" (2014-2015), exhibit atypically high levels of correlation with many of the other criteria. We can explain this with the varying sets of countries in these years. Another basic requirement, " 2 Infrastructure", also often appears to strongly correlate with another economic enhancers, "9 Technological readiness". This is a 
noteworthy finding in the context of researching these intercriteria relations on the background of countries in transition from factor-driven to efficiency-driven economy.

\section{Conclusions}

The two considerations about the essence of the analyzed dataset in this leg of the ICA research on GCR data, about the size of the analyzed set, and its elements' variability over time, are important to weigh up in discussing the results and their reliability and comparability to prior results of similar ICA applications. From the results in Tables 8 to 12 and Figure 1, is seen that the majority of the intercriteria pairs are in the dissonance zone, and despite the relatively low uncertainty (lower in the beginning, and higher in the end of the period) the pairs of criteria with relatively similar values of membership and non-membership are not conclusive for more categorical decisions on this basis, for the countries in the transition from factor- to efficiencydriven economy. These results, however, can obtain a better interpretation after we complete the research of all global economies, and we are able to compare the performance of the pillars and the dependences between them within these different contexts, and over time.

\section{Acknowledgements}

The Bulgarian authors are grateful for the support provided under Grant Ref. No. DN-02-10 of the Bulgarian National Science Fund.

\section{References}

[1] Atanassov, K., Andonov, V., Krawczak, M. (2017) On intuitionistic fuzzy modes, medians and mean elements. Notes on Intuitionistic Fuzzy Sets, 23(3), 17-22.

[2] Atanassov, K., Atanassova, V., Szmidt, E., \& Kacprzyk, J. (2018) Intuitionistic Fuzzy Interpretations of Some Formulas for Estimation of Preference Degree. In: Soft Computing Applications for Group Decision-making and Consensus Modeling, Springer, Studies in Fuzziness and Soft Computing, Cham, Vol. 357, 153-161.

[3] Atanassov, K., Mavrov, D., \& Atanassova, V. (2014) InterCriteria decision making: a new approach for multicriteria decision making, based on index matrices and intuitionistic fuzzy sets. Issues in Intuitionistic Fuzzy Sets and Generalized Nets, 11, 1-8.

[4] Atanassov, K., Szmidt, E., \& Kacprzyk, J. (2013) On intuitionistic fuzzy pairs. Notes on Intuitionistic Fuzzy Sets, 19(3), 1-13.

[5] Atanassov, K., Szmidt, E., Kacprzyk, J., \& Atanassova, V. (2017) An approach to a constructive simplification of multiagent multicriteria decision making problems via intercriteria analysis. Comptes rendus de l'Academie bulgare des Sciences, 70(8), 1147-1156.

[6] Atanassova, V. (2015) Interpretation in the intuitionistic fuzzy triangle of the results, obtained by the InterCriteria Analysis, Proc. of 16th World Congress of IFSA, 9th Conf. of EUSFLAT, 30. 06-03. 07. 2015, Gijon, Spain, 2015, 1369-1374. 
[7] Atanassova V., Doukovska, L., Atanassov, K., \& Mavrov, D. (2014) InterCriteria decision making approach to EU member states competitiveness analysis, Proc. of the International Symposium on Business Modeling and Software Design - BMSD'14, 2426 June 2014, Luxembourg, Grand Duchy of Luxembourg, 289-294.

[8] Atanassova, V., Doukovska, L., Kacprzyk, A., Sotirova, E., Radeva, I., \& Vassilev, P. InterCriteria analysis of the Global Competitiveness Reports: from efficiency- to innovation-driven economies. Journal of Multivalued Logic and Soft Computing. (accepted).

[9] Atanassova, V., Doukovska, L., Karastoyanov, D., \& Capkovic, F. (2014) InterCriteria decision making approach to EU member states competitiveness analysis: Trend analysis. P. Angelov et al. (eds.), Proc. of IEEE Intelligent Systems'2014, Advances in Intelligent Systems and Computing, 322, Springer, 107-115.

[10] Atanassova, V., Doukovska, L., Mavrov, D., \& Atanassov, K. (2014) InterCriteria decision making approach to EU member states competitiveness analysis: Temporal and threshold analysis. P. Angelov et al. (eds.), Proc. of IEEE Intelligent Systems'2014, Advances in Intelligent Systems and Computing, 322, Springer, 95-106.

[11] Doukovska, L., Atanassova, V., Sotirova, E., \& Traneva, V. (2018) Knowledge discovery from the Global Competitiveness Reports: Intercriteria analysis of the efficiency-driven economies. Notes on Intuitionistic Fuzzy Sets, 24(3) (in print).

[12] Doukovska, L., Atanassova, V., Sotirova, E., Vardeva, I., \& Radeva, I. (2019) Defining Consonance Thresholds in InterCriteria Analysis: An Overview. In: Intuitionistic Fuzziness and Other Intelligent Theories and Their Applications, Springer, Studies in Computational Intelligence, Vol. 757, DOI: 10.1007/978-3-319-78931-6_11 (in print).

[13] Ikonomov, N., Vassilev, P., \& Roeva, O. (2018) ICrAData - Software for InterCriteria Analysis, Int J Bioautomation, 22(1), 1-10.

[14] Mavrov, D. (2015) Software for InterCriteria Analysis: Implementation of the main algorithm, Notes on Intuitionistic Fuzzy Sets, 21(2), 77-86.

[15] Mavrov, D. (2015-2016) Software for InterCriteria Analysis: Working with the results. Annual of "Informatics" Section, Union of Scientists in Bulgaria, 8, 37-44.

[16] Roeva, O., Vassilev, P., Angelova, M., Su, J., \& Pencheva, T. (2016) Comparison of different algorithms for intercriteria relations calculation. Proc. of IEEE 8th Int. Conf. on Intelligent Systems, Sofia, 4-6 September 2016, 567-572.

[17] Roeva, O., P Vassilev, P., \& Chountas, P. (2017) Application of Topological Operators over Data from InterCriteria Analysis, Lecture Notes on Artificial Intelligence, Vol. 10333, FQAS 2017, 215-225.

[18] Schwab, K. (2013) The Global Competitiveness Report 2013-2014. World Economic Forum, Geneva, ISBN-13: 978-92-95044-73-9.

[19] Schwab, K. (2014) The Global Competitiveness Report 2014-2015. World Economic Forum, Geneva, ISBN-13: 978-92-95044-98-2. 
[20] Schwab, K. (2015) The Global Competitiveness Report 2015-2016. World Economic Forum, Geneva, ISBN-13: 978-92-95044-99-9.

[21] Schwab, K. (2016) The Global Competitiveness Report 2016-2017. World Economic Forum, Geneva, ISBN-13: 978-1-944835-04-0.

[22] Schwab, K. (2017) The Global Competitiveness Report 2017-2018. World Economic Forum, Geneva, ISBN-13: 978-1-944835-11-8.

[23] Todorova, L., Vassilev, P., \& Surchev, J. (2016) Using Phi Coefficient to Interpret Results Obtained by InterCriteria Analysis, In: Novel Developments in Uncertainty Representation and Processing, AISC, 401, Springer, 231-239.

[24] Zoteva, D., \& Roeva, O. (2018) InterCriteria Analysis results based on different number of objects. Notes on Intuitionistic Fuzzy Sets, 24(1), 110-119. 